\title{
ON TORSIONLESS SUBGROUPS OF INFINITELY GENERATED FUCHSIAN GROUPS
}

\author{
PEKKA TUKIA
}

A very useful property of finitely generated Fuchsian groups is that such a group always contains a normal subgroup of finite index with no elliptic elements. In this paper we prove an analogous result for infinitely generated Fuchsian groups. Obviously, in this case we cannot always find a torsionless subgroup of finite index but it turns out that a very pleasant analogy exists. We also determine when there exists a torsionless normal subgroup of finite index.

Let $G$ be a finitely generated Fuchsian group. Essentially, all proofs of the fact that $G$ contains a normal subgroup of finite index without elliptic elements use the following method. One can find a finite group $F$ and a homomorphism $\varphi: G \rightarrow F$ such that $\varphi^{-1}(e) \subset G$ is torsionless ( $e$ is the neutral element of $F$ ). Then $N=\varphi^{-1}(e)$ is necessarily of finite index in $G$ and does not contain elliptic elements. In $[2,3,5]$ and [9, Chapter IV.10] the proof was based on a description of $G$ in terms of generators and relations. In contrast, Selberg [6] considered $G$ as a subgroup of the group of real or complex $n \times n$ matrices. Thus the theorem is, in fact, true for all finitely generated matrix groups, and also for Kleinian groups. Originally, the theorem for finitely generated Fuchsian groups was a conjecture of W. Fenchel.

Our method is in spirit like the first method, and it is based on the results of $[7,8]$, especially on the fact that if a Fuchsian group has a non-compact orbit space, then $G$ is a free product of finite or infinite cyclic groups. If it were possible to generalize Selberg's method, we would have results for countable matrix groups.

We need not assume that the elements of the groups we are considering are orientation preserving. Therefore, in the following Theorem, a Fuchsian group is a discrete group of orientation preserving or reversing Möbius transformations of $U=\{z \in C: \operatorname{Im} z>0\}$. The limit set of a Fuchsian group $G$ is denoted by $L(G)$. Then $L(G) \subset R \cup\{\infty\}$.

Theorem. Let $G$ be a Fuchsian group such that $G$ does not contain reflections and that $U / G$ is non-compact. Then there is a torsionless normal subgroup $N \subset G$ such that:

(i) If $g \in G$, there is $n>0$ such that $g^{n} \in N$.

(ii) If $H \subset G$ is a finitely generated subgroup, then $N \cap H$ is a torsionless normal subgroup of $H$ that is of finite index in $H$. 
(iii) $L(G)=L(N)$.

(iv) The group $G$ contains a subgroup of finite index without elliptic elements if and only if the orders of elliptic elements of $G$ are bounded. In this case we may assume that the index of $N$ in $G$ is $m$ where $m>0$ is the smallest number divisible by the order of every elliptic element of $G$.

Remark 1. Let $N^{\prime} \subset G$ be a torsionless subgroup of index $m^{\prime}<\infty$. If $g \in G$ is elliptic and if $k$ is the order of $g$, then $k$ divides $m^{\prime}$. Thus, if $m$ is as in (iv), $m^{\prime}$ is divisible by $m$.

Proof. Let $E(G)=\{z \in U$ : thẹre is $g \in G \backslash\{$ id $\}$ with $g(z)=z\}$. Then, by [8, Proposition p.15], $G$ has a fundamental domain $D$ such that $\operatorname{bd}_{U} D \backslash E(G)\left(\mathrm{bd}_{U}=\right.$ boundary in $U$ ) is a disjoint union of hyperbolic lines or hyperbolic rays from which the endpoint in $U$ has been removed. Let $C_{i}, i \in I$, denote these lines and rays, i.e. $\operatorname{bd}_{U} D \backslash E(G)=\left(\bigcup_{i \in I} C_{i}\right) \backslash E(G)$. By the same proposition, there are $g_{i} \in G$ for each $i \in I$ such that $g_{i}\left(C_{i}\right)=C_{j}$ for another $j \in I, j \neq i$, and that $g_{i}^{-1}=g_{j}$ if $g_{i}\left(C_{i}\right)=C_{j}$. In addition, if $C_{i}$ is a hyperbolic ray, then $g_{i}\left(C_{i}\right)$ is another ray such that $C_{i} \cap g_{i}\left(C_{i}\right)$ is the fixpoint of $g_{i}$ in $U$.

Let $J \subset I$ be a subset such that, for each $i \in I$, exactly one of the sets $C_{i}$ and $g_{i}\left(C_{i}\right)$ is in $\left\{C_{j}: j \in J\right\}$. Let $Z_{i}, i \in J$, be the cyclic subgroup generated by $g_{i}$. Now it is easy to see that $G$ is the free product

$$
G=\underset{i \in J}{*} Z_{i},
$$

which is also proved in [7, the proof of Theorem 2.6]. Let $g \in G$ be elliptic and let $z \in U$ be the fixpoint of $g$. Then there is $h \in G$ such that $h(z) \in D$, i.e. $h(z)$ is the fixpoint in $U$ for some $g_{i}, i \in J$. Then we must have $g=h^{-1} g_{i}^{k} h$ where $0<k<$ the order of $g_{i}$.

Now we define a map $\varphi: G \rightarrow Q / Z$ as follows. If $i \in J$ and $Z_{i}$ is infinite, let $\varphi\left(g_{i}\right)=0$. If the order of $Z_{i}$ is $n_{i}<\infty$, let

$$
\varphi\left(g_{i}\right)=\text { class of } 1 / n_{i} \text { in } Q / Z \text {. }
$$

This defines $\varphi$ uniquely. If $g \in G$ is elliptic, there is, as indicated above, $h \in G$ such that $h g h^{-1}=g_{i}^{k}$ for some $i \in J$ and $0<k<n_{i}$. Then

$$
\varphi(g)=\text { class of } k / n_{i} \neq 0 \text { in } Q / Z \text {. }
$$

Thus $N=\varphi^{-1}(0)$ does not contain elliptic elements. We will show that $N$ satisfies conditions (i)-(iv).

Clearly, $N$ satisfies (i). Let $H \subset G$ be finitely generated. Then $\varphi(H)$ is finite and therefore $N \cap H$ is of finite index in $H$. Thus (ii) is true. Since $L(G)=$ $\operatorname{cl}\{x \in R \cup\{\infty\}: x$ is a fixpoint of some element of $G\}$, and (i) is true, also (iii) must be true. By (2) we have that $\varphi(G)$ is the subgroup of $Q / Z$ which is generated by \{class of $1 / k \in Q / Z: k$ is the order of some elliptic element of $G$ \}. Thus $N$ is of finite index if the orders of elliptic elements of $G$ are bounded, and in this case the index of $N$ in $G$ is the number $m$ of (iv). By the above Remark 1, a subgroup $N^{\prime} \subset G$ 
cannot be of finite index unless the orders of elliptic elements of $G$ are bounded. Thus (iv) is true.

Remark 2. Above we have referred to [7] and [8] for the proof of the fact that $G$ is of the form (1). This proof is unnecessarily long if one wishes to have only this result. A shorter proof can be obtained by rewriting the proof in Section 44A of Ahlfors-Sario [1, Ch. I], i.e. one chooses a canonical exhaustion $K_{0} \subset K_{1} \subset \ldots$ for $S=U / G$, a base point $x_{0} \in K_{0}, \tilde{x}_{0} \in U$ projecting to $x_{0}$, and lets $G_{i}$ be the subgroup of $G$ obtained by lifting closed loops in $K_{i}$ beginning at $x_{0}$ to paths beginning at $\tilde{x}_{0}$. Then one shows that $G_{i}$ is a finite free product of cyclic groups and that $G_{i+1}$ is a free product of $G_{\imath}$ and cyclic groups.

We have also used the fact that an element of $G$ of finite order is conjugate to an element of some $Z_{i}$ in (1). This follows also from the Kurosh subgroup theorem (cf. e.g. Massey [4, Theorem 5.1]), and we could have deleted the beginning of the above proof since by [7, Corollary 2.8.1] $G$ is of the form (1). However, we have preferred the above geometric proof to the use of the Kurosh subgroup theorem.

The above mentioned proof of Ahlfors-Sario [1, I. 44A] that the fundamental group of an open Riemann surface is free, was pointed out to me by T. Erkama.

\section{References}

[1] Ahlfors, L. V., and L. SARio: Riemann surfaces. - Princeton University Press, Princeton, New Jersey, 1960.

[2] Bundgaard, S., and J. Nielsen: On normal subgroups with finite index in F-groups. - Mat. Tidsskr. B. 1951, 56-58.

[3] Fox, R. H.: On Fenchel's conjecture about F-groups. - Mat. Tidsskr. B. 1952, 61-65.

[4] Massey, W. S.: Algebraic topology: An introduction. - Harcourt, Brace \& World, Inc., New York-Chicago-San Francisco-Atlanta, 1967.

[5] Nielsen, J.: The commutator group of the free product of cyclic groups. - Mat. Tidsskr. B. 1948, 49-56 (Danish).

[6] SelberG, A.: On discontinuous groups in higher-dimensional symmetric spaces. - Contributions to function theory, (Internat. Colloq. Function Theory) Tata Institute of Fundamental Research, Bombay, 1960, 147-164.

[7] Tukia, P.: On discrete groups of the unit disk and their isomorphisms. - Ann. Acad. Sci. Fenn. Ser. A I 504, 1972, $1-45$.

[8] Tukia, P.: Extension of boundary homeomorphisms of discrete groups of the unit disk. - Ann. Acad. Sci. Fenn. Ser. A I 548, 1973, 1-16.

[9] Zieschang, H., E. Vogt, and H.-D. Coldewey: Flächen und ebene diskontinuierliche Gruppen. - Lecture Notes in Mathematics 122, Springer-Verlag, Berlin-HeidelbergNew York, 1970.

University of Helsinki

Department of Mathematics

SF-00100 Helsinki 10

Finland

Received 22 June 1978 